Cahiers $d u$ MONDE RUSSE

\section{Cahiers du monde russe}

Russie - Empire russe - Union soviétique et États indépendants

$52 / 4 \mid 2011$

Varia

\title{
Stephen V. Bittner, The Many Lives of Khrushchev's Thaw
}

\section{Bella Ostromoukhova}

\section{OpenEdition \\ Journals}

Édition électronique

URL : http://journals.openedition.org/monderusse/7636

DOI : $10.4000 /$ monderusse.7636

ISSN : $1777-5388$

\section{Éditeur}

Éditions de l'EHESS

\section{Édition imprimée}

Date de publication : 20 décembre 2011

Pagination : 780-785

ISBN : 978-2-7132-2353-2

ISSN : $1252-6576$

Référence électronique

Bella Ostromoukhova, «Stephen V. Bittner, The Many Lives of Khrushchev's Thaw », Cahiers du monde russe [En ligne], 52/4 | 2011, mis en ligne le 03 décembre 2012, Consulté le 22 septembre 2020. URL http://journals.openedition.org/monderusse/7636 ; DOI : https://doi.org/10.4000/monderusse.7636

Ce document a été généré automatiquement le 22 septembre 2020

(c) École des hautes études en sciences sociales 


\title{
Stephen V. Bittner, The Many Lives of Khrushchev's Thaw
}

\author{
Bella Ostromoukhova
}

\section{RÉFÉRENCE}

Stephen V. BITTNER, The Many Lives of Khrushchev's Thaw. Experience and Memory in Moscow's Arbat. Ithaca - London : Cornell University Press, 2008, xi - 235 p.

1 Le livre de Stephen V. Bittner est centré sur les diverses façons de vivre et de conceptualiser, au moment même du vécu et a posteriori, l'époque de Hruščev, considérée à la fois dans son altérité et sa continuité avec l'avant stalinien et l'après brejnévien. L'originalité de l'approche que propose Bittner pour appréhender cette période dernièrement fort étudiée réside dans le choix d'un support géographique concret, celui du quartier moscovite de l'Arbat. On aurait tort, toutefois, de guetter des emprunts méthodologiques à la géographie humaine ou à la sociologie urbaine qui mèneraient, par exemple, à une étude des dynamiques de peuplement de ce quartier, des différentes façons de vivre et d'occuper l'espace particulier de l'Arbat, ou encore des interactions entre les politiques d'urbanisme et les riverains. Même si ces questions sont implicitement disséminées dans l'ouvrage, elles n'en constituent toutefois pas le centre. Il s'agit plutôt d'une étude assez classique d'histoire culturelle : l'auteur met un coup de projecteur sur quelques institutions situées dans l'Arbat, ce qui lui permet de plonger dans des débats caractéristiques du dégel.

2 L'Arbat offre, en effet, un terrain propice à une telle approche. Comme le souligne Bittner dans le premier chapitre, "histoire et mythe de l'Arbat ", ce quartier a été associé - et l'est toujours, d'une certaine manière - à l'intelligentsia. Proche du centre, l'Arbat abritait depuis le $\mathrm{XIX}^{\mathrm{e}}$ siècle une population importante d'artistes et d'intellectuels, ainsi qu'un certain nombre d'institutions à vocation culturelle comme, par exemple, l'Institut supérieur de musique des sœurs Gnesin, créé en 1895, le théâtre Vahtangov logé depuis 1920 au 26 rue de l'Arbat, ou encore l'Institut de recherche sur 
la littérature mondiale (IMLI) de l'Académie des sciences soviétique qu'abritait un hôtel particulier de la rue Povarskaja depuis la fin des années 1930. Cette concentration d'artistes et d'intellectuels a induit la naissance d'un «mythe » de l'Arbat, mis en forme et porté, entre autres, par l'écrivain Anatolij Rybakov et le poète et chanteur Bulat Okudžava. Les deux mêlent dans leurs œuvres l'histoire du quartier à leur expérience individuelle : l'Arbat aurait connu un âge d'or dans les années 1920 et au début des années 1930, époque de leur enfance, qui s'achèverait dans les répressions de la fin des années 1930. Le quartier serait donc devenu la métaphore de l'intelligentsia créatrice qui fut détruite par Stalin et ne reviendra plus jamais. Zone piétonne depuis le milieu des années 1980, la rue de l'Arbat, artère principale du quartier, serait aujourd'hui emblématique de cette disparition: attirant des populations "étrangères " à ses habitants (représentants de cultures alternatives, marchands de souvenirs, touristes), la rue serait devenue un musée en plein air où toute vie quotidienne normale serait impossible. Un monument à Bulat Okudžava, qui s'élève à l'intersection de la rue de l'Arbat et de la ruelle Plotnikov, aurait réussi à représenter cet "esprit de l'Arbat " évanescent. Le poète est bien là, incarnant l'« arbatstvo » dont il se réclamait, mais il est aussi en train de s'en aller, de quitter « la cour de l'Arbat » symbolisée par deux arches, présente dans ses chansons à la fois comme lieu de jeux de son enfance et comme marque de noblesse.

3 Cette dégradation de l'esprit de l'Arbat aurait déjà commencé, pour Okudžava comme pour Rybakov, pendant l'époque du dégel, ce qui permet à Bittner de relativiser la manière dont cette époque avait été vécue par les contemporains. Comme il l'affirme en introduction, la manière dont l'intelligentsia avait vécu l'ère Hruščev était très différente des représentations qu'elle en avait faites a posteriori, notamment après les procès des dissidents de la seconde moitié des années 1960. Dans la nouvelle éponyme d'Ehrenbourg, le dégel n'évoquerait pas l'épanouissement printanier qu'y verraient le plus souvent les universitaires et les témoins oculaires, mais bien plutôt un réchauffement fugace et incertain au milieu de l'hiver. Loin de susciter un enthousiasme unanime, le dégel était souvent vécu comme une période ambiguë, pleine de contradictions, et à l'issue incertaine. L'exemple concret des institutions culturelles situées dans l'Arbat ainsi que celui des débats liés à la reconstruction du «Nouvel Arbat » permettent à l'auteur de montrer comment l'intelligentsia serait passée d'une méfiance circonspecte à l'enthousiasme, puis à la déception et, enfin, à la nostalgie.

4 L'Institut Gnesin, étudié en détail dans le deuxième chapitre, permet à Bittner de montrer les nombreuses contradictions qui animaient l'univers de la musique. Depuis les années 1940, le domaine de l'histoire et de la théorie de la musique avait été structuré par le jdanovisme dont le décret du Comité central du parti, daté de 1948, sur "l'opéra de Vano Muradeli La grande amitié » marquait un point culminant. Après la mort de Stalin, et plus encore après la dénonciation de son "culte de la personnalité " lors du XXe congrès du parti en 1956, il était devenu évident, pour les enseignants de l'institut, qu'ils devaient réécrire les manuels d'histoire et de théorie de la musique et élargir le spectre des sujets abordés en cours. Ainsi, notamment, la musique occidentale contemporaine devait y être étudiée, d'autant plus que les étudiants, curieux de nouveautés, le réclamaient. Toutefois, les enseignants se heurtaient à une ambiguïté de la part des autorités : alors qu'une révision des programmes était exigée, le décret de 1948 n'était pas révoqué et le «formalisme " musical était toujours officiellement réprouvé. Les limites du permis étaient donc fluctuantes au cours du temps, comme l'étaient également les convictions des enseignants eux-mêmes. Bittner fournit des 
éléments biographiques qui permettent d'expliquer ces revirements. Ainsi, certains de ces enseignants avaient eu leurs conjoints réprimés par Stalin, eux-mêmes avaient été visés par les campagnes contre le « cosmopolitisme». La prudence les incitait donc à soutenir les postulats du jdanovisme jusqu'à quelques mois après le $\mathrm{XX}^{\mathrm{e}}$ congrès. Toutefois, cette attitude les opposait aux étudiants qui y voyaient une marque de complicité avec le stalinisme. À l'automne 1956, quelques mois après la dénonciation des crimes de Stalin, ces mêmes enseignants ont commencé à se prononcer en faveur d'une révision radicale du jdanovisme. Les autorités ne leur en ont donné l'autorisation sans équivoque qu'en 1958, en révoquant enfin le décret de 1948. Cette contradiction, entre la nécessité de réviser les postulats idéologiques et l'impossibilité de le faire, était loin d'être unique. Ainsi, par exemple, alors que les étudiants de l'Institut Gnesin étaient de plus en plus encouragés à participer aux concours internationaux, les soulèvements en Pologne et en Hongrie en 1956 avaient incité les autorités de l'institut à surveiller l'exposition des étudiants à l'influence étrangère.

5 L'univers de la création théâtrale était, lui aussi, pétri de contradictions que Bittner esquisse dans le troisième chapitre, à partir de l'exemple du théâtre Vahtangov. Ayant connu son heure de gloire dans les années 1920, cet établissement s'était toutefois trouvé à l'écart de l'effervescence théâtrale du dégel. Pour le sortir de cette impasse, le metteur en scène Simonov, ancien disciple de Vahtangov, a décidé de recréer la production de la Princesse Turandot, opéra de Gozzi qui a fait la gloire du théâtre au début des années 1920. Le spectacle original, élaboré au sein du Studio du théâtre de l'art dirigé par Vahtangov, était porteur d'idées d'avant-garde : il reprenait la toute première version de l'opéra, écrit pour la commedia dell'arte, qui portait des traces du théâtre de rue et où l'improvisation jouait un grand rôle. Ponctué d'anachronismes, le spectacle renvoyait le spectateur au présent, le forçait à adopter un rôle actif. En reprenant ces éléments en 1962, Simonov entendait non seulement rendre un hommage à ces procédés de l'avant-garde des années 1920, mais également réanimer l'atmosphère au sein de la troupe en réintroduisant des éléments de studijnost' ${ }^{\prime}$. Le succès de cette tentative restait mitigé : «l'innocence, l'esprit égalitaire et l'inventivité juvénile du studio de Vahtangov» (p.104) pour lesquels Simonov et ses collègues ressentaient de la nostalgie, ne pouvaient être revécus. En revanche, la perception du rôle du pouvoir avait changé depuis l'époque stalinienne: la tentative du pouvoir d'imposer au théâtre la pièce de Vojtehov, L'orage, clairement antistalinienne mais jugée de mauvaise qualité par les metteurs en scène et les acteurs, a suscité une indignation au sein de la troupe et s'est terminée par une aporie.

Les chapitres quatre et cinq offrent une incursion dans le domaine de l'architecture et concernent l'événement qui a radicalement transformé la physionomie du quartier dans les années 1960, à savoir la construction de l'artère du Nouvel Arbat. Entre 1962 et 1968, un grand nombre d'immeubles anciens avaient été détruits pour laisser la place à une rue "moderne ", bordée de gratte-ciels et de centres commerciaux. Le quatrième chapitre montre que ce projet s'était situé à la confluence entre, d'une part, la volonté de Hruščev de promouvoir des projets de construction «type " (typovoe proektirovanie) bon marché afin de répondre à une crise du logement et, d'autre part, le désir de certains architectes du MOSA (l'Union moscovite des Architectes) de réhabiliter le constructivisme honni pendant la période stalinienne. Du pragmatisme du pouvoir et de la nostalgie pour les années 1920 des architectes est né le projet du Nouvel Arbat comme une artère du futur, dont les gratte-ciels abriteraient des bureaux ou des logements et hôtels de luxe pour hauts fonctionnaires. Ce chantier qui a marqué la fin 
de l'époque Hruščev, débordant sur l'ère Brežnev, a suscité des mouvements de protestation, notamment - et c'est le sujet du cinquième chapitre du livre - de la part des partisans du mouvement "Préservation du patrimoine architectural», qui commençait à prendre de l'ampleur pendant l'époque du dégel. Les tentatives de sauver des bâtiments historiques se sont révélées le plus souvent vaines. Toutefois la manière de procéder des militants marquait une différence par rapport à l'ère stalinienne : les défenseurs du patrimoine architectural n'adressaient pas des motions au pouvoir pour demander la sauvegarde de tel ou tel monument, mais exigeaient que la population soit consultée lors des destructions massives. L'opinion publique devait, selon ces militants, conjointement à l'avis des professionnels, faire autorité en matière de jugement de valeur dans le domaine du patrimoine architectural. Ce rôle de l'opinion publique aurait été rendu possible par les espoirs de changement que le dégel aurait finalement fait naître parmi les intellectuels.

7 Espoirs déçus dès le milieu des années 1960, notamment par suite des procès des dissidents dont le sixième chapitre et dernier chapitre du livre dissèque de près le contexte. L'IMLI, l'Institut de recherches sur la littérature mondiale, situé également dans le quartier de l'Arbat, était le lieu de travail d'Andrej Sinjavskij depuis 1952. Julij Daniel', lui, n'y travaillait pas, mais avait « de nombreux amis parmi les chercheurs et les doctorants ». Depuis la mort de Stalin, l'institut avait connu plusieurs réformes qui visaient à diminuer le degré d'ingérence du parti dans le travail scientifique et à donner le droit aux chercheurs de fixer leurs propres objectifs de travail et d'en évaluer les résultats indépendamment des impératifs de l'Académie des sciences. Cette nouvelle indépendance a généré un climat d'effervescence intellectuelle, les chercheurs se sont tournés vers des sujets bannis auparavant - la littérature des années 1910-1920 ainsi que les œuvres étrangères - attirant ainsi l'attention des autorités, internes et externes, qui remettaient en cause la toute fraîche et fragile autonomie des chercheurs. L'affaire Sinjavskij et Daniel', accusés d'avoir publié à l'étranger des œuvres antisoviétiques, a failli perturber cet équilibre de forces précaire. Le Comité central du parti avait exigé qu'une lettre collective des collaborateurs de l'institut dénonçant les crimes de Sinjavskij soit publiée dans la presse et que, par ailleurs, le secrétaire local du parti, tenu pour responsable de l'indiscipline idéologique du chercheur, soit remplacé. Aucun de ces deux ordres n'a été obéi. Même si les collègues de Sinjavskij et Daniel' n'ont pas essayé ouvertement de sauver les deux hommes de lettres, ils ont réussi à préserver une relative liberté qui a servi de cadre aux actions dissidentes. Actions qui ont trouvé une large répercussion dans le monde intellectuel, montrant que certains effets du dégel, aussi fugace fût-il, étaient permanents.

Ainsi, le quartier de l'Arbat et les représentations qui y sont liées offrent à l'auteur l'occasion d'aborder, d'une manière très concrète et détaillée, les différentes manières dont le dégel marquait, tout au long des années 1950 et 1960, les multiples microcosmes de la création artistique et de la recherche. Oscillant entre contexte général et cas très particuliers, foisonnant de détails personnels, le livre offre de nombreuses pistes de réflexion, mais donne également lieu à de nombreuses digressions dont on a parfois du mal à saisir le lien avec le sujet principal. Par ailleurs, les personnages historiques sont décrits avec une précision qui leur donne de la chair mais qui peut également irriter. Ainsi, par exemple, le contexte de rivalité entre le metteur en scène Simonov et un autre disciple de Vahtangov, longuement exposé dans le deuxième chapitre, permet au lecteur de mieux comprendre la volonté de recréer La princesse Turandot. On peut toutefois se demander s'il est vraiment important, par exemple, de savoir que Simonov 
était « un guitariste avéré et un fan de football qui parfois annulait des répétitions pour assister aux matchs de son équipe préférée, le Spartak»(p.86). D’autres détails confèrent au récit un ton tragique étrange pour un ouvrage scientifique. Ainsi, l'hôtel particulier qui abrite l'IMLI est présenté comme « associé à une tragédie » depuis plus d'un siècle (p. 174) car il avait été acheté en 1875 par l'époux de la fille aînée de Puškin, Leonid Gortung, qui s'était donné la mort deux années plus tard par suite d'un scandale public. Sans rapport avec les faits relatés, ce détail cité en début de chapitre sur les dissidents pourrait faire croire qu'un « destin » néfaste planait sur ce lieu, supposition qui aurait plus sa place dans une histoire de fantômes que dans un livre d'histoire.

Ces digressions montrent la volonté de l'auteur de rendre moins austère l'exercice académique. Intention qui est visible dans la structure même du livre qui prend la forme d'une promenade dans le quartier dont les bâtiments livrent leur histoire. Plaisante, cette flânerie laisse toutefois le lecteur sur sa faim : on regrette que l'Arbat ne soit finalement qu'un prétexte pour écrire l'histoire de l'intelligentsia créatrice. On peut se demander si le lien entre les personnalités décrites et le quartier était réel ou si l'auteur, tout en soulignant l'aspect mythologique du destin de l'Arbat, ne s'est pas finalement laissé gagner lui-même par le mythe. L'Arbat a-t-il été pour Sinjavskij ou pour Simonov autre chose que leur adresse professionnelle? Aucun élément, dans l'ouvrage, ne permet de répondre à cette question. Un autre aspect du mythe, celui du lien inextricable entre le quartier et l'intelligentsia, pousse l'auteur à identifier ces deux éléments, au point d'ignorer la mixité sociale de l'Arbat qu'il mentionne toutefois en introduction. On regrette que la population ordinaire qui habitait ce quartier pendant le dégel n'apparaisse que très ponctuellement, au détour d'une phrase, et que la parole ne lui soit jamais donnée alors que l'auteur mentionne, sans les citer, des "témoignages des riverains" concernant, notamment, les difficultés de la vie quotidienne actuelle. Ces habitants non illustres de l'Arbat auraient également le droit à ce que leur « expérience et [leur] mémoire » contribuent au récit des «multiples vies du dégel ».

\section{NOTES}

1. Terme parfois traduit en français par « esprit de studio », notion centrale pour les collectifs en marge des troupes professionnelles comme l'était, au départ, le studio de Vahtangov. Cet « esprit » supposait une cohésion très forte du groupe, cimentée par l'unité spirituelle de ses membres totalement désintéressés économiquement, entièrement dévoués à l'entreprise commune de renouvellement des formes théâtrales et soumis à une discipline de fer. 